\title{
Development of comprehensive unattended child warning and feedback system in vehicle
}

\author{
Norizam Sulaiman ${ }^{1, *}$, Kamarul Hawari Ghazali ${ }^{1}$, Mohd Shawal Jadin ${ }^{1}$, Amran Abdul Hadi ${ }^{1}$, \\ Muhammad Sharfi Najib ${ }^{1}$, Mohd Salmizan Mohd Zain ${ }^{1}$, Fatimah Abdul Halim ${ }^{1}$, \\ Suhaimi Mohd Daud ${ }^{1}$, Nurdiyana Zahed ${ }^{1}$ and Abdul Adam Abdullah ${ }^{2}$ \\ ${ }^{1}$ Faculty of Electrical \& Electronics Engineering, Universiti Malaysia Pahang, 26600 Pekan, Pahang, \\ Malaysia \\ ${ }^{2}$ Faculty of Mechanical Engineering, Universiti Malaysia Pahang, 26600 Pekan, Pahang, Malaysia
}

\begin{abstract}
The cases of children being trapped and suffocated in unattended vehicle keep increasing even though the awareness campaign on the safety of children in non-moving vehicle were carried out by government. Various methods were introduced by researchers to overcome this issue but yet to be effective. Among them were the usage of capacitive sensor, microwave sensor, pressure sensor and image sensor where most of the techniques or systems were applied on the child's seat to detect the presence of baby or infant. Thus, this research is carried out to provide a comprehensive and effective detection system to detect the presence of children including infant in unattended vehicle by using the combination of human physiological signals (voice and body odor) detectors with the temperature and motion sensors. Here, once the proposed system recognizes any signals that generated from voice, odor, motion and temperature detectors in vehicle's cabin, the system then will provide effective feedback system by sending short message to the parents first. If no response received in the specified allocation time, the system then will activate the vehicle's horn system. Finally, the system will lower down the vehicle's window to release the toxic gas and reduce the cabin temperature. The system is in prototyping stage where every design component was evaluated individually. Besides, the overall system was successfully tested where the detection and feedback system follow the instruction given by the microcontroller.
\end{abstract}

\section{Introduction}

In Malaysia, vehicle-related deaths for children fewer than 12 years of age keep occurring and the cases are in increasing trend [1-2]. Despite of various inventions to enhance the vehicle safety system and awareness campaign to prevent those incidents from occurring, the deaths caused by the non-moving vehicle keep increasing. When the car engine is turned-off with closed window, the temperature in the car will increase rapidly and children

* Corresponding author: norizam@ump.edu.my 
might suffer heat stroke [1, 3]. A child's body overheat more easily than an adult's [1]. Most of the incidents occurred due to the negligence of the parents [2-4]. Parents or drivers, in certain situations, may leave the car without taking the children. It might also have occurred when the father or mother change his or her duty to send their children to nursery. This scenario can happen since their children taking sleep or nap, and they might not realize the presence of their children in the vehicle especially for the bigger vehicle such as multipurpose vehicle which has more than two rows of seats. Unlike vehicle in United States or European countries which requiring parents to put their children in the specified child's seat before driving as a safety matter, those rules are not abide by most of the Malaysian vehicle's drivers. It seems that the requirement to use child's seat is applied merely on the children with age below 2 years old. However, children with age ranging from 2 to 6 years old are free to move around inside the vehicle's cabin. Thus, they will be sitting, laying or napping somewhere in the vehicle's cabin. For example, it will be very hard for the multipurpose vehicles' owner to locate his or her child if the child is lie down at the back seat or at the floor between the second and last row of the seat. It is a human nature to forget something and to think driving alone. As a result, he or she will park and leave the vehicle while his or her child is left behind or still inside the vehicle [5].

Various techniques and invention had been introduced for couple of decade but yet to provide the solid solution for to cater this issue. Most of the techniques and invention were patented especially in United States and Europe. In United States and Europe, the child detection systems were developed especially for baby or infant seat and integrated with car alarm system. Here, the car security system will generate an audio alarm once the car is turned-off and the children are detected still in the car baby seat [4-5]. Among the various child detectors were introduced by researchers are Passive Infrared Red (PIR) motion sensor, RFID, RF safety pad, capacitive sensor, microwave sensor, pressure transducer, temperature detector and image detector. However, all the invention is still yet to provide the effective and comprehensive warning and feedback systems to trigger parent for the unattended child in the vehicle. Some of the inventions have drawbacks. For example, for the child seat, there were synchronization issues between child-seat-sensing device and notification device. Meanwhile, PIR required motion from the children and will not effective for the children in sleep condition [6-8]. Another invention was the use of pressure transducer on the child seat to detect the changing of air pressure due to the opening and closure of the vehicle door [9]. Here, the device was integrated with the vehicle alarm system to produce alarm once the pressure transducer activated. In addition, one of the invented techniques was the combination of the child seat sensor and temperature detector were applied to detect the present of child and to detect unsafe temperature in the vehicle cabin and trigger the vehicle alarm system [10]. The drawback for both inventions is they are designed purposely for infant which required to be placed onto child seat to trigger the car alarm system. Meanwhile, for image detection technique, it requires wide angle camera and of course, a lot of camera are required for bigger vehicle such as multi-purpose vehicle or sport utility vehicle. Another method is the use of visual cue at the car dashboard to enable driver to have a view behind the driver seat. However, this method might not practicable for bigger vehicle such as multipurpose vehicle.

In term of communication systems, it cannot be denied that the wireless communication is a practical and an effective medium to enable human to communicate with electronics device. Among them are IEEE 802.4.15, the network Global System for Mobile Communications (GSM), Global Positioning System (GPS) and Radio Frequency Identification system (RFID) were used extensively especially in vehicle safety technology [11-16]. In current vehicle technology, most of the modern vehicle already equipped with wireless anti-theft security system whereas the child detection system is installed on the vehicle baby seat and strictly applied for vehicle in US and European countries. In those 
countries, there is a law to put the young child or infant in the child seat when driving the vehicle [15-16]. In Asian countries especially Malaysia, the law is not fully followed and merely the expensive vehicle has the accessories for anti-theft and child detection system. Thus, it a must for every vehicle in Malaysia regardless the type of the vehicle, to be equipped with the wireless child detection system since the system is proven by researchers to enable human to communicate with the vehicle regardless the location of the vehicle unless the location cannot be reached by the GSM.

Therefore, this research will introduce a comprehensive child detection system which enhancement of the previous researches by combining human physiological signals such as voice and odor with image, temperature and motion detection systems. Besides, the system will also provide the efficient feedback system if any failure on alarm system or no response from the parent on the notification system through GSM module or no response from people who might think that the alarm sound come from the faulty alarm. The system then will be integrated with the existing vehicle security and ignition systems. The proposed system is constructed to achieve the followings objective; to design a warning system to detect the presence of children in unattended vehicle using a combination of voice, odour, motion, and temperature detection techniques. Besides, the system is created to provide the intelligent feedback system by sending Short Messaging System (SMS) to the parents, activating car's horn system and finally, moving down the vehicle's window in order to release the toxic gas and reduce the temperature inside the vehicle's cabin.

\section{Experimental setup}

This section will describe the overall process in designing, developing and testing the proposed system

\subsection{Design of experiment}

The design process begins by selecting the suitable input signals, signals processor and types of effective feedback signals. Hence, the research is divided into three studies; detection system, feedback system and selection of microcontroller to process the input signals and provide the good triggering system as illustrated by Fig. 1. In this study, 4 input signals are chosen; temperature, motion, voice and odor. In order to detect those signals, the separate detectors or recognizers are constructed. For example, temperature detector is developed to measure interior temperature of the vehicle and detect any changes in interior temperature in vehicle's cabin after the vehicle being parked. Arduino-based temperature sensor is used which can measure temperature up to $125^{\circ} \mathrm{C}$. Meanwhile, motion detector is constructed using a passive infrared (PIR) sensor which become a top choice of detectors by researchers in this study due to the high accuracy, easy handling and low cost. Voice detector is developed by using the combination of Adafruit FONA mini GSM (SIM900L), EasyVR Shield and Arduino Uno R3. Here, Adafruit is used to send text message to the mobile phone through GSM module. SIM900L is a complete Quad-band GSM/GPRS in a SMT type. Meanwhile, EasyVR Shield is used to capture the voice and Arduino acts as a microcontroller to process the voice that received from the shield. In order to increase the accuracy of detecting voice, about 9 voices from difference child are captured and processed by the microcontroller. 


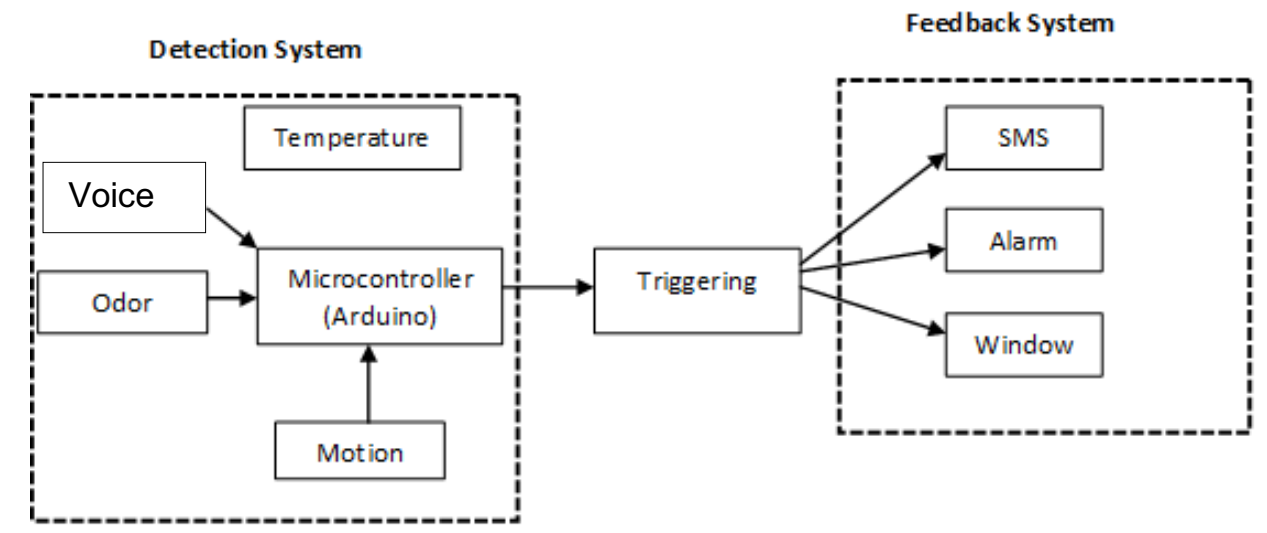

Fig. 1. Block diagram of the detection and feedback system.

The most challenging task in this research is to design the odor recognizer. Here, the odor recognizer consists of E-Nose [17]. E-nose is made up by a chamber equipped with sensor array and can be linked to microcontroller. The sample will be fed at the inlet part of the chamber and the output will be released at the outlet part of the chamber. It is a device invented by one of the member in research team to detect multiple type of odor. The ENose was employed to classify the Kelulut (one of bee species) honey. The intelligent detection and classification system is developed to study the odor profile and determine the type of odor as illustrated in Fig. 2. In this research, the odor of child's urine and sweat is captured and collected from 9 children. Then, the profile of those odors is created and studied. Also involved in the study is the odor from vehicle's perfume and cabin. The study requires much time in order to obtain the correct odor profile that come from human (child). The thorough study on child's odor profile is implemented to ensure the correct instruction is given to the microcontroller for the feedback system.

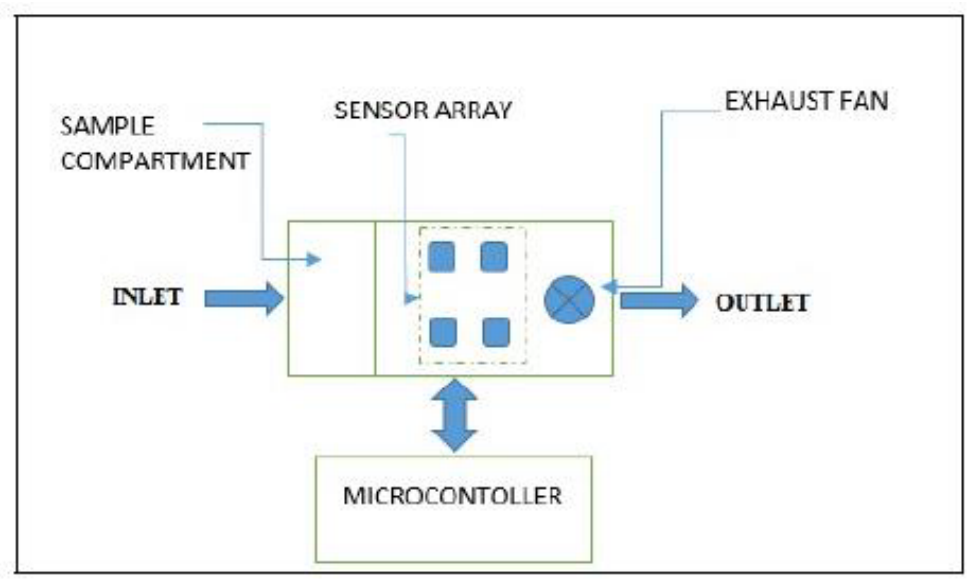

Fig. 2. Block diagram of odour detection system. 


\subsection{Operation of the proposed system}

The proposed system will improvise the previous system by adding human physiological signals and creating the smart feedback system to detect the presence of children in the unattended vehicle. As shown by block diagram in Fig. 1, the project covers the design of the detection and feedback system which include the testing of various sensors. Hence, the experimental produce will include the chronological sequences of the system in detecting the input signals from the sensors and triggering the feedback system.

The system starts in five minutes after the vehicle is in its parking situation. The vehicle is considered to be in parking situation based on several conditions such as ignition engine is central lock is on or handbrake is locked or ignition engine is off. The alert voice message will be sent to the vehicle driver about the activation of the detection and feedback system. The system then will activate the temperature sensor first, follow by motion, voice and odor detectors respectively. However, to make the system more robust, in real implementation of the project, any detector activated will trigger the feedback system either by sending SMS to vehicle driver, activating vehicle's horn and rolling down the vehicle's window. Here, the temperature sensor will activate when the cabin temperature greater than $40^{\circ} \mathrm{C}$. Meanwhile, the voice and motion detectors will take place to activate the system if detecting any movement or voice from the child. The child will start to cry or move his or her body when feeling uncomfortable due to the increasing cabin temperature. In order to make the detection system more comprehensive and since the system will take time to react based on the triggering condition of the temperature, motion and voice sensors, odor recognizer is introduced. Here, once the vehicle in its parking position with engine off, the odor detector is activated. It can immediately detect the present of the child in the vehicle's cabin based on the odor profile of the child without waiting for the other sensors to be activated. Once the odor of the child is detected, the system will trigger the feedback system immediately. The feedback system will begin by sending SMS to the vehicle driver's mobile phone through the GSM module. If no response from the vehicle driver, the vehicle horn system is activated. Finally, if no response from the vehicle driver, the feedback system will move down the window at little bit just to reduce the cabin's temperature and release the toxic gas. The system need to be reset manually by the vehicle's driver.

The full schematic of the circuit design of project is depicted by Fig. 3. As described by the diagram, 2 units of Arduino microcontroller are used to process the multiple input signals. Based on the schematic shown in Fig. 3., LCD is used to display the alert message when detecting motion and vehicle interior's temperature. The digital pins of LCD (D4, D5, D6 and D7) are connected to the digital pin of Arduino 1 (D9, D10, D11 and D12). The E and RS pins of LCD are connected to Arduino D7 and D8. Here, the temperature sensor is connected to the analog pin of Arduino (A5). Meanwhile, the transmitter and receiver pins of Adafruit GSM module is connected to the receiver and transmitter pins of Arduino Uno R3 (Digital pin 1, D1 and Digital pin 0, D0). The window motor is connected to the relay pins (GS1, GS2, GS3 and GS4). Then, the relay pins are connected to the analog pins of Arduino (A0, A1, A2 and A3). In addition, motion sensor is connected to the Arduino digital pin D6. Meanwhile, odor and voice recognizer's input signals are connected to the analog pins of another Arduino (Arduino 2). The second Arduino is attached to the EasyVR shield. The LED lights (red, yellow and green) are also installed into the detection system to indicate the status of detection process. 


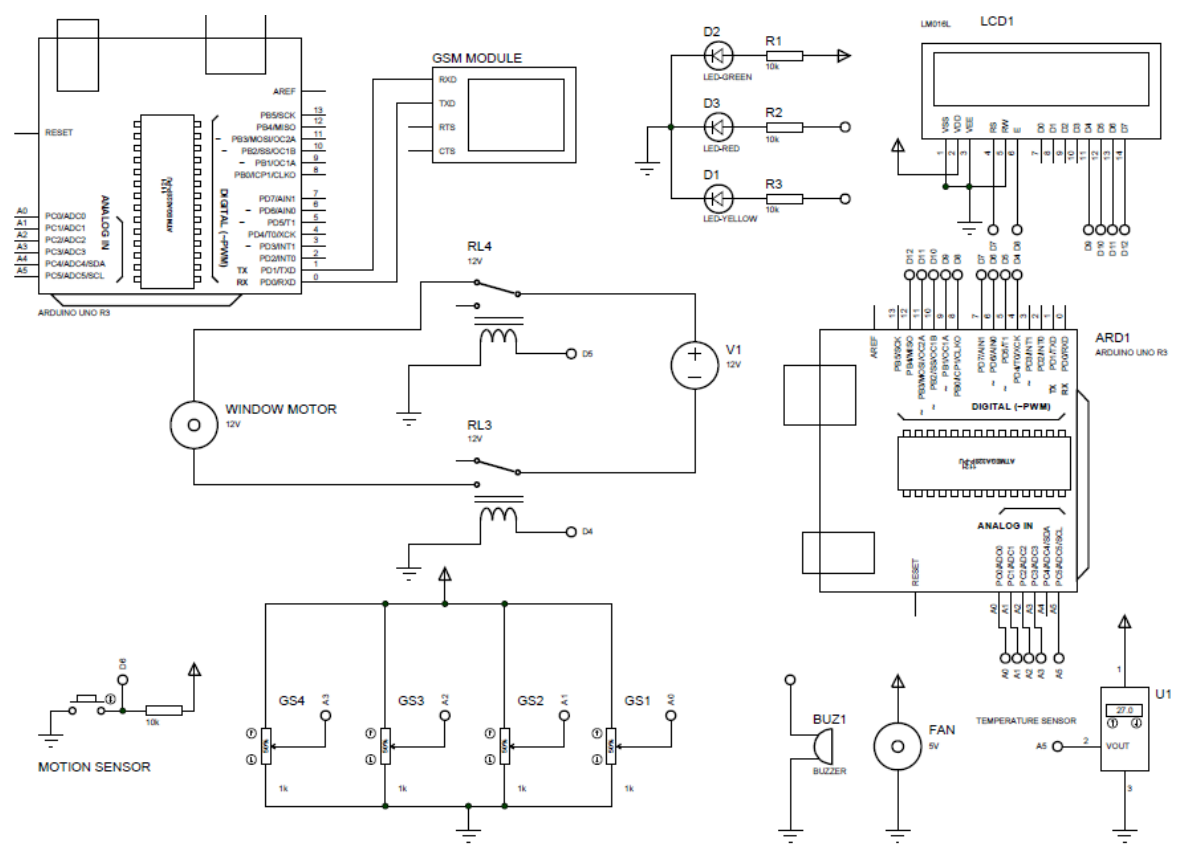

Fig. 3. The full schematic of the detection and feedback system.

\section{Results and discussion}

The project consists of designing the detection and feedback system using multiple detectors; temperature, motion, voice and odor. The temperature, motion and voice detection system is developed using the temperature sensor, PIR sensor and voice recognizer. Meanwhile, odor recognizer is built using E-Nose. Then, all the sensors are assembled and tested for functionality and accuracy. The prototype of temperature and motion detection system is shown in Fig. 4. When PIR detect any motion or temperature sensor detects the increasing surrounding temperature, the signals will be sent to Arduino microcontroller to process it, and then the results will be displayed on the LCD. The microcontroller also will send the processed data to the feedback system to trigger the vehicle driver by activating the alarm system.

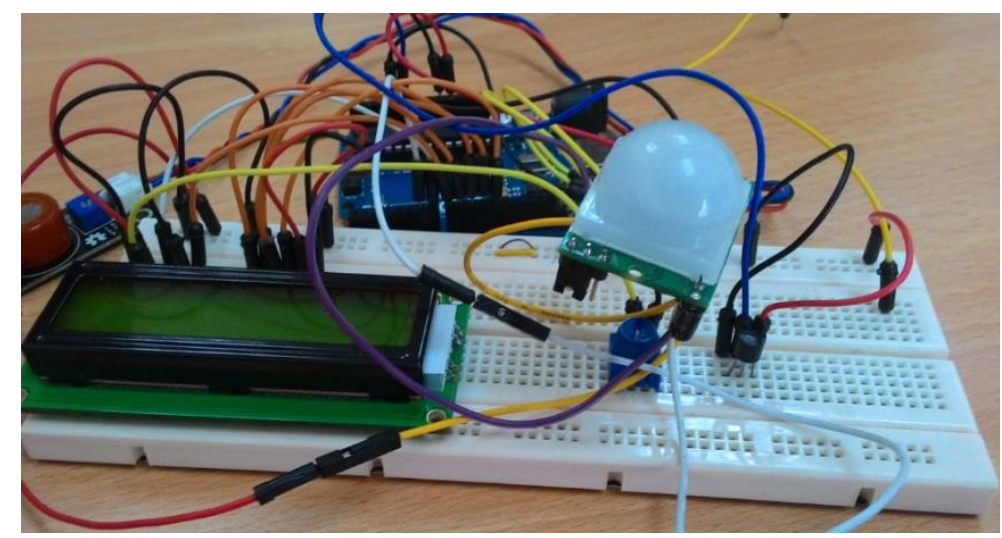

Fig. 4. Temperature and motion recognizer. 
Meanwhile, the odor detection system is built using chamber and sensor array. The odor recognizer is tested for accuracy with various odor profiles including profile of child's odor before assembling it with the other sensors. The training on the odor recognizer needs to be implemented in order to enhance the capability of that recognizer to differentiate and distinguish human's odor from other source of odor in the vehicle's cabin. The odor recognizer should able to recognize the child's odor accurately based on the child's urine and sweat. Once the odor is confirmed come from child, the detection system will trigger the feedback system.

The voice detection system is constructed using Adafruit FONA mini GSM, voice recognizer shield (EasyVR) and Arduino. Also, attached to the voice recognizer shield is the microphone to activate the system. The system is tested with 9 different child's voice and able to recognize all the voice accurately. Once the system able to recognize the child's voice, it will trigger the feedback system by sending SMS to mobile phone, activate car horn and rolling down the vehicle's window. The prototype of the voice detection system is shown in Fig 5. The example of the SMS sent to the mobile phone is elucidated by Fig. 6 .

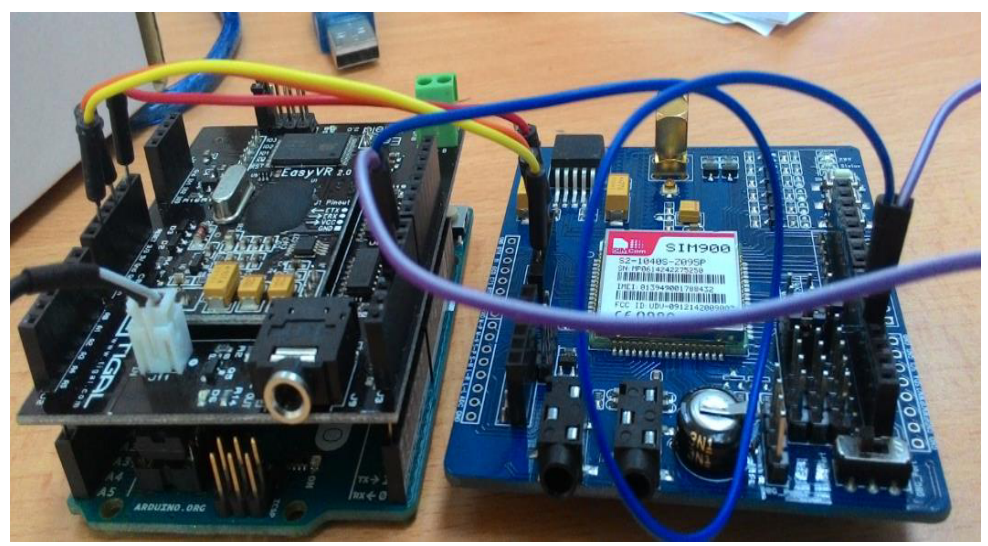

Fig. 5. Voice recognizer.

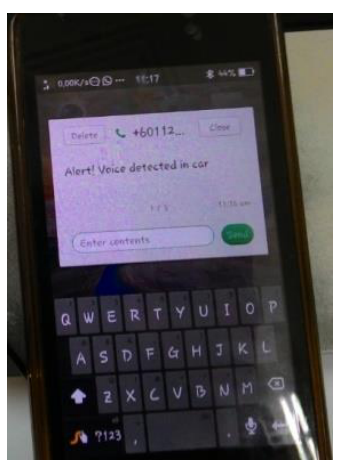

Fig. 6. Feedback system through Short Message (SMS).

The overall prototype of the detection and feedback system is elucidated by Fig. 7 (a). The prototype consists of $12 \mathrm{~V}$ battery to provide voltage to window motor, bigger black box consists of temperature, motion, odor sensors, LCD, LEDs and Arduino microcontroller. Meanwhile, the smaller black box consists of EasyVR shield, Adafruit FONA mini GSM, Arduino microcontroller, microphone and antenna. During testing the prototype, the Arduino microcontroller is power-up through USB cable that connected to the computer. The direction of the window motor either rotating clock wise or counter 
clock wise and duration of rotation will depend on the instruction given to the microcontroller. The location of window motor in the vehicle's door is shown in Fig. 7 (b).

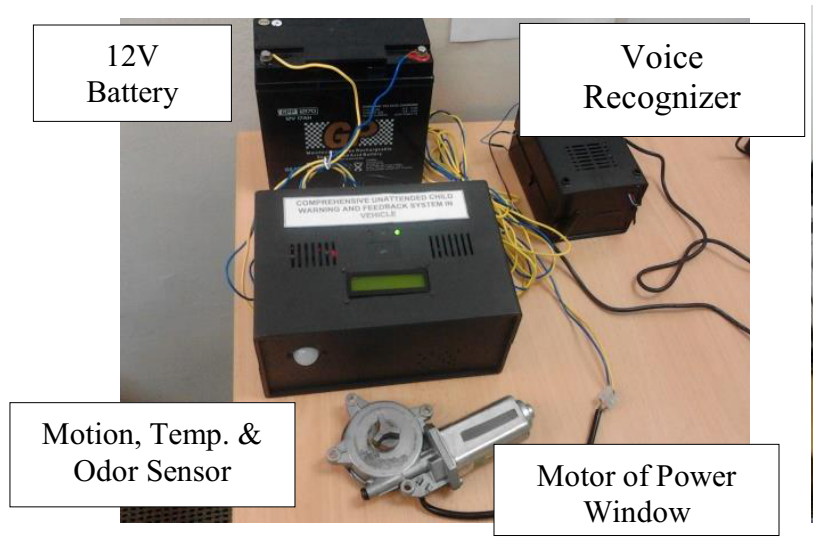

(a)

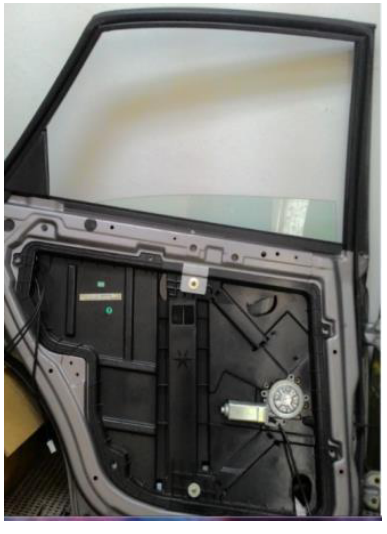

(b)

Fig. 7. The overall prototype of detection and feedback system.

\section{Conclusions}

In this paper, the prototype of the detection and feedback system is constructed successfully. All the sensors were tested individually and functioning correctly according to the instruction given by microcontroller. The main advantage of the system compared to the existing system is the usage of odor recognizer based on E-nose technology. The outcome of the study shows that the use of human physiological signals (voice and odor) is very effective to detect the present of the child in the unattended vehicle. The future work may include enhancement of the system by using smaller prototype such as using printed circuit board (PCB). Therefore, it can be fit nicely inside the vehicle's cabin. The next project will also include the integration of the proposed system with the vehicle's electronic system. The robustness testing of the system might be implemented as well.

The authors would like to express high appreciation to the postgraduate students and research team members for their great assistance and support during the project implementation and testing. The authors are also grateful to Universiti Malaysia Pahang for providing financial assistance through research grant, RDU140376.

\section{References}

1. Consumer Report News (2012)

2. F.R.M. Rashidi, I.H. Muhamad, Recent Advances in Automation, Control, Modelling and Simulation (2013)

3. A. Ranjan, B. George, Instrumentation and Measurement Technology Conference (2013)

4. J. Booth, G. Davis, J. Waterbor, G. McGwin, Forensic Science, Medicine, and Pathology, 6, (2010)

5. M. Rossi, U.S. Patent 5949340 (1999)

6. N. M. Z. Hashim, H. H. Basri, A. Jaafar, M. Z. A. A. Aziz, A. Salled, A. S. Ja'afar, ARPN Journal of Engineering and Applied Sciences 9, 9 (2014) 
7. J. Morningstar, U.S. Patent 7714737 B1 (2007)

8. I., H. Muhamad, F.R.M Rashidi, Proceeding of the Recent Advances in Automatic Control, Modelling and Simulation (2013)

9. M. G. Burgess, U.S. Patent 6812844 B1 (2004)

10. D. C. White, U.S. Patent 7701358 B1 (2010)

11. K. Vidyasagar, G. Balaji, K. Narendra Reddy, International Journal of Communications on Applied Electronics 2, 2 (2015)

12. J. K Lee, D. R. Spach, P. Srinivasan, D. P. Umstead. U.S. Patent 7321306 B2 (2008)

13. G. C. Kautz, I. La, J. Beach, U.S. Patent 34957-6605 (2010)

14. F. Mesina, IEEE Journal (2005)

15. J. Abdullah, International Journal of Engineering and Technology 3 (2011)

16. K. N. Kamil, S. I. A. Rahman, M. Gambilok, ARPN Journal of Engineering and Applied Sciences 10, 22 (2015)

17. M. S. Najib, S. H. Azih, N. Zahed, M. F. Zahari, W. M. A. Mamat, H. Manap, ARPN Journal of Engineering and Applied Sciences 11, 10 (2016) 